\title{
Correlative Light and Electron Microscopy for Materials Science
}

\author{
Jessica L. Riesterer ${ }^{1}$ and Gregor Heiss ${ }^{1}$ \\ ${ }^{1 .}$ FEI Company, Hillsboro, OR, USA.
}

Although a powerful approach, correlative light and electron microscopy (CLEM) for materials and life sciences has been historically challenging because of low throughput. Providing full morphological information with respect to chemistry and topography is the strength of electron microscopy. Fluorescence microscopy excels at labeling components with unmatched sensitivity and specificity; however, it lacks contextual information and has limited resolution. By imaging the same sample with both imaging modalities it is possible to combine the advantages and overcome the limitations of the individual microscopy techniques. ${ }^{1}$

FEI has recently introduced new solutions to simplify the CLEM workflow, and in turn, increase the experimental throughput; CorrSight ${ }^{\mathrm{TM}}$, a dedicated light microscope providing CLEM-specific functionality and automation of important workflow steps with MAPSTM, a software tool bridging the modalities to increase ease of use and provide time savings by automating the data collection process. When coupled with SEM, FIB, and additional imaging modalities, ${ }^{2}$ these tools address different correlative workflows helping to optimize efficiency and data quality across the full range of CLEM experiments.

Ruby $\left(\mathrm{Al}_{2} \mathrm{O}_{3}\right.$ with trace $\left.\mathrm{Cr}\right)$ in fuchsite $\left(\mathrm{K}(\mathrm{Al}, \mathrm{Cr})_{3} \mathrm{Si}_{3} \mathrm{O}_{10}(\mathrm{OH})_{2}\right)^{3}$ was imaged using both FEI's CorrSight and Helios NanoLab 660 DualBeam ${ }^{\mathrm{TM}}$. Ruby has long been known to fluoresce ${ }^{4}$ due to the trace amounts of $\mathrm{Cr}$ in solid solution. Using the Helios' through-lens detector (TLD) in BSE-mode coupled with the MAPS software, extreme low-voltage high-resolution data was collected over large areas. The use of $\mathrm{Cu}$ tape on the specimen edges serves two purposes: enhances conductivity to reduce charge and serves as a rough fiducial marker to assist in correlation and navigation.

Following BSE imaging, the fluorescent properties of specific regions were investigated using the CorrSight and correlated BSE data. In figures 1 and 2, red in the fluorescence image corresponds to blue light, while blue indicates signal collected via reflection. The CorrSight, used to obtain reflective polarized light microscopy images, was equipped with a 20/80 beam splitter and excitation and emission polarizer. The excitation light of $480 \mathrm{~nm}$ was linearly polarized and reflected to the sample surface via a beam-splitter. The reflected light from the surface was redirected to the emission polarizer through the same beam-splitter. However, the emission beam-splitter was cross-polarized with respect to the excitation polarizer to enhance the signal from bi-refringent properties of the crystal.

Once the instrumentation is correlated using a simple two-point alignment in MAPS, fracture planes, inclusions, and secondary phases can be identified quickly and easily by comparing imaging modalities. Correlated images can be used to identify $\mathrm{Cr}$-rich regions and ruby inclusions much faster than EDS mapping over large surface areas. Correlation also aids in narrowing the search for particular elementaldependent events. The demonstrated workflow is shown to be viable over large regions of interest, i.e. several millimeters, in an automated way, leading to significant time savings. 


\section{References:}

[1] M.A. Snyder, et al, Micropor. Mesopor. Mat. 76 (2004), p. 29

[2] B. Van Leer and R. Passey, $18^{\text {th }}$ International Microscopy Conference, Prague, ISBN 978-80-2606720-7 (2014) p. 181

[3] http://www.mindat.org/min-1617.html

[4] C. S. Venkateswaran, Proc. IISc. 2 (1935), p. 459.

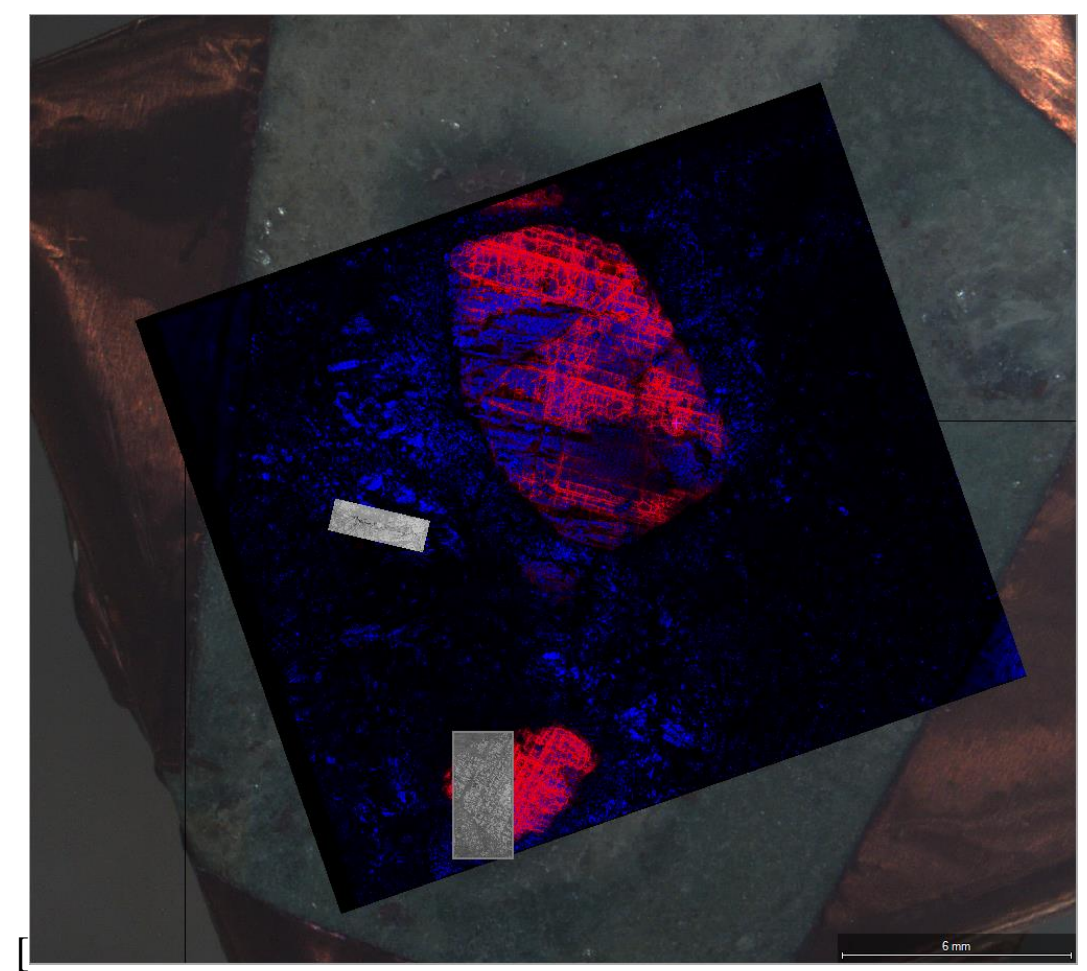

Figure 1. Three imaging modalities, visible-light, fluorescence, and SEM, can be easily correlated using the MAPS workflow. Black scale bar is equal to $6 \mathrm{~mm}$.
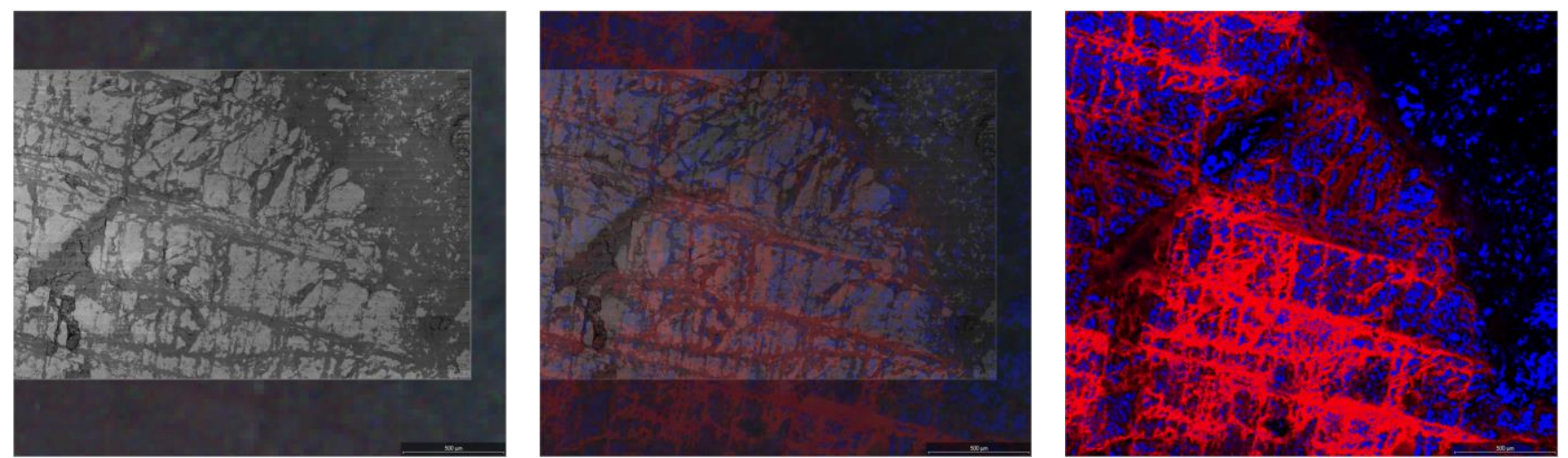

Figure 2. By overlaying (center) data from SEM (left) and LM (right), fine structure and second-phase inclusions can be quickly identified. Black scale bar is equal to $600 \mu \mathrm{m}$. 